\title{
Sustained Remission in Tumor Necrosis Factor Inhibitor-treated Patients with Rheumatoid Arthritis: A Population-based Cohort Study
}

\author{
Jon Thorkell Einarsson, Pierre Geborek, Tore Saxne, and Meliha C. Kapetanovic
}

\begin{abstract}
Objective. To study frequency, possible baseline predictors, timing, and duration of sustained remission [SR; defined as 28-joint Disease Activity Score (DAS28) $<2.6$ for at least 6 mos] in patients with established rheumatoid arthritis (RA) treated with different tumor necrosis factor (TNF) inhibitors [etanercept (ETN), infliximab (IFX), adalimumab (ADA)]. In addition, the aim was to compare (head-to-head) the effectiveness of individual drugs in patients receiving their first anti-TNF treatment.

Methods. All anti-TNF-treated patients with RA included in the observational South Swedish Arthritis Group register were eligible. We identified the patients' first SR periods (time between first visit after treatment initiation with DAS2 $8<2.6$ and subsequent visit with DAS28 $\geq 2.6$ ). Baseline predictors of SR in biologic-naive patients were studied using multivariate regression models. Remission duration and timing of remission start was estimated with Kaplan-Meier curves.

Results. Of the 2416 patients included, 382 (15.8\%) fulfilled the criteria for SR. Median estimated duration of SR was 5.25 years. Predictors for SR were male sex, low Health Assessment Questionnaire, low DAS28, methotrexate (MTX) treatment, and the calendar year of treatment start. OR for achieving SR within the first 12 months of treatment were 1.86 for ETN (95\% CI 1.33-2.61) compared to IFX. HR for 4 years of SR were 1.32 for ETN (95\% CI 1.01-1.74) and 1.84 for ADA (95\% CI 1.23-2.78), with IFX as the reference drug.

Conclusion. SR was uncommon in patients with RA treated with anti-TNF in clinical practice. However, patients remained in SR for a substantial period of time. Concomitant MTX treatment predicts remission. ETN and ADA were more likely in reaching SR. (First Release Feb 15 2015; J Rheumatol 2015;42:741-8; doi:10.3899/jrheum.131502)
\end{abstract}

Key Indexing Terms:

RHEUMATOID ARTHRITIS REMISSION

TNF INHIBITORS

REGISTER

BIOLOGIC TREATMENT PREDICTORS
Rheumatoid arthritis (RA) is associated with significant morbidity, mortality, and cost for society ${ }^{1,2,3,4}$. During the last 2 decades, there have been major changes in the treatment and management of RA, including earlier intervention with disease-modifying antirheumatic drugs (DMARD), aggressive dosing, combination therapy, and the introduction of new classes of therapeutic agents ${ }^{5}$.

From the Department of Clinical Sciences Lund, Section of Rheumatology, Lund University, Skåne University Hospital, Lund, Sweden.

J.T. Einarsson, MD; P. Geborek, MD, PhD; T. Saxne, $M D, P h D$;

M.C. Kapetanovic, MD, PhD, Department of Clinical Sciences Lund, Section of Rheumatology, Lund University, Skåne University Hospital.

Supported by grants from the Swedish Rheumatism Association, the Swedish Research Council, the Medical Faculty of Lund University, the Alfred Österlund Foundation, the Crafoord Foundation, the Greta and Johan Kock Foundation, King Gustaf V's 80-Year Foundation, and Lund University Hospital.

Address correspondence to Dr. J.T. Einarsson, Department of Clinical Sciences Lund, Section of Rheumatology, Skåne University Hospital, Lund University, Kioskgatan 3, SE-221 85 Lund, Sweden.

E-mail: jonthorkell@gmail.com

Accepted for publication January 2, 2015.
Since the introduction of biologic agents targeting anti-tumor necrosis factor (TNF) as a treatment option in the late 1990s, remission of disease activity has become the goal of treatment ${ }^{6,7}$. No universally accepted remission criteria exist, although an American College of Rheumatology (ACR)/European League Against Rheumatism (EULAR) collaboration has resulted in a new criteria set for remission in patients with $\mathrm{RA}^{8}$. However, these criteria do not account for the time aspect because being in remission is more important if it is sustained over a period of time ${ }^{9,10}$.

The objective of our present study was to investigate frequency, possible baseline predictors, timing, and duration of sustained remission [SR; i.e., 28-joint Disease Activity Score (DAS28) < 2.6 for at least 6 mos] in patients with established RA starting anti-TNF treatment with etanercept (ETN), infliximab (IFX), or adalimumab (ADA) in daily clinical practice. In addition, the aim was to compare (head-to-head) different anti-TNF medications with regard to frequency, duration, and timing of SR in patients receiving their first anti-TNF treatment.

Personal non-commercial use only. The Journal of Rheumatology Copyright @ $\subset$ 2015. All rights reserved. 


\section{MATERIALS AND METHODS}

Data were retrieved from the South Swedish Arthritis Treatment Group register (SSATG), a large, prospective, observational study cohort involving 11 rheumatology clinics. The registry contains detailed disease severity characteristics at baseline and at followup for patients treated with biologic drugs dating from $1999^{11}$. All data entries are centralized to ensure uniform interpretation of registration forms. Several validation studies have proved it to be population-based for patients with RA with coverage of pharmacy sales of $>90 \%^{12,13}$. In Sweden, the government is the only buyer of healthcare. During the study period, the treating physician had no restrictions for selecting biologic therapy and was not required to taper treatment during remission.

All patients with an RA diagnosis according to the clinical judgment of the treating physician and who were starting anti-TNF treatment with ETN, IFX, or ADA during the period from March 1999 through December 2009 were eligible for the study. A previous review of patients included in this cohort showed that $98 \%$ of the patients fulfilled the 1987 ACR classification criteria for $\mathrm{RA}^{14}$.

The decision to initiate anti-TNF treatment was based on disease activity and/or unacceptable glucocorticoid use. No formal level of disease activity was required; however, the patients should have received at least 1 DMARD previously without acceptable response. The registry was searched for individual treatments fulfilling the DAS28 remission criteria on at least 2 consecutive occasions $\geq 3$ months after treatment initiation. One researcher (JTE) scrutinized every treatment case by case, ensuring true DAS28 remission. Remission time was defined as the time between first visit with DAS $28<2.6$ and subsequent first visit with DAS28 $\geq 2.6$. To compensate for intercurrent diseases, patients were allowed to have DAS28 $\geq 2.6$ on 1 occasion given that they fulfilled the remission criteria for at least 6 months before and after. These patients were categorized as having SR. Only the first treatment with SR per patient was analyzed, i.e., individual patients only contributed with $1 \mathrm{SR}$ treatment. However, patients could have had previous biologic treatment with no SR, i.e., stopped because of adverse event, primary failure, or other stop reason. Followup data was collected through June 2010 or treatment termination/switch to another biologic. No data imputation was done.

Between March 1999 and December 2009, 3446 treatments with anti-TNF were initiated in 2416 patients with RA. In these 2416 patients, their first treatment used for analysis was 1034 ETN (43\%), 937 IFX $(39 \%)$, and $445 \mathrm{ADA}(18 \%)$. The majority of the analyzed treatments (95.6\%) were in biologic-naive patients (Figure 1). Mean followup time from treatment start to either treatment stop or study stop was 40 months (range 0-149).

Statistical analyses. Demographic, disease, and patient characteristics at the start of anti-TNF therapy were calculated in all patients and also after stratification according to reaching remission, i.e., never remission, non-sustained, and SR.

The frequency of SR in all patients and for each individual drug were calculated. Time to and duration of SR were also calculated.

Possible baseline predictors of SR in patients on first biologic treatment were studied using multivariate regression models. Predictors chosen based on clinical relevance included sex, age, disease duration (per year increase), C-reactive protein (CRP), DAS28, Health Assessment Questionnaire (HAQ) scores, concurrent glucocorticoid use (yes/no), concurrent methotrexate (MTX) use (yes/no), and the calendar year of treatment start (1998-2007). Possible colinearity between variables was assessed by the Spearman correlation test. Separate models were used for DAS28 status and HAQ status because DAS28 and HAQ showed high levels of colinearity (Spearman rho $r=0.47$ ). However, all the other variables were included in the final multivariate analysis. The results from the HAQ model are presented as OR with $95 \%$ CI.

Survival analysis (Kaplan-Meier survival curve) was used for estimating remission duration for all anti-TNF treatments and the first biologic treatment for each individual drug. Kaplan-Meier estimates included response rates using per protocol, intention to treat (ITT) using last observation carried forward, and the LUNDEX calculations to account for patients who had withdrawn from treatment. LUNDEX is an index combining the proportion of patients fulfilling a selected response criteria set (in this case, SR) with the proportion of patients adhering to a particular therapy (in this case, drug survival):

estimated fraction responding at time $\mathrm{T} \times$ estimated fraction of starters still in the study at time $\mathrm{T}$

LUNDEX correction was used to provide ITT data in the observational setting. This is an established method for giving balanced response data in the observational setting with incomplete followup visits ${ }^{15}$. LUNDEX correction corresponded to randomized controlled trials (RCT) nonresponder imputation.

For drug comparison only, first anti-TNF treatment was analyzed for a given patient and 4-year followup time was used as a cutoff point because ADA was not available in clinical practice until 2004.

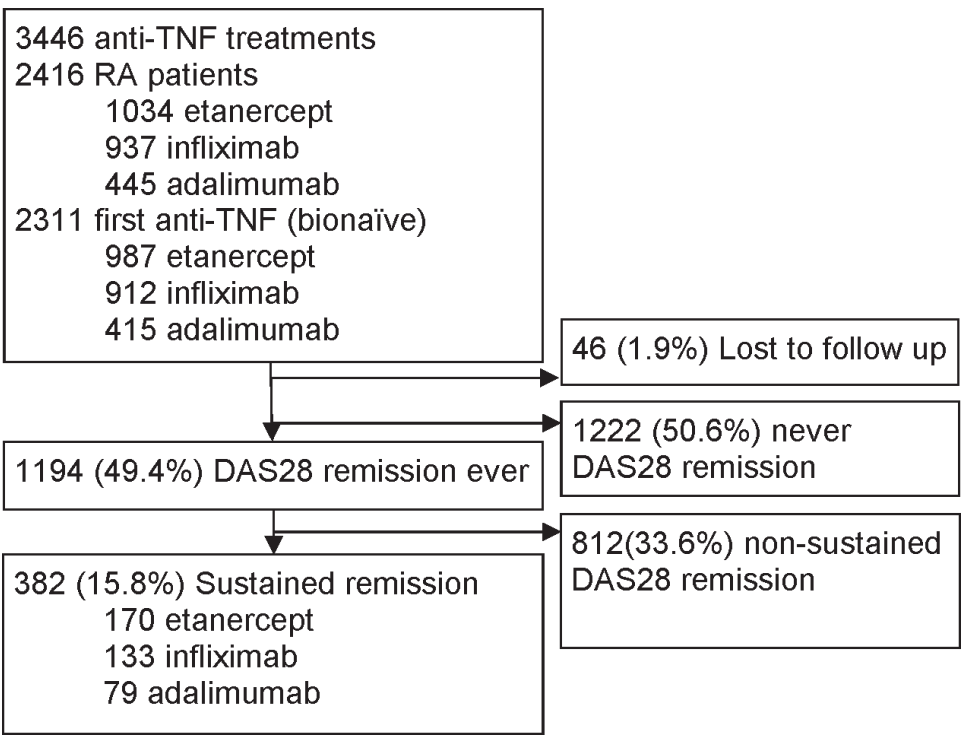

Figure 1. Study design and population. TNF: tumor necrosis factor; RA: rheumatoid arthritis; DAS28: 28joint Disease Activity Score. 
SR of individual drugs at 12 months after treatment start were analyzed using multivariate logistic regression. Results are given as OR. The probability for ever reaching SR was analyzed using Cox regression and probability for remission survival before escape (secondary failure). Results are given as HR. Lost to followup or treatment termination for any other reason (e.g., remission, pregnancy, adverse event) was censored. Possible confounders, each added one by one to the raw model, were tested. In case of significant change of regression coefficient $(\geq 10 \%)$, the variable was included in the final model. Possible confounders included age, sex, disease duration, prednisolone (PSL) use (yes/no), MTX use (yes/no), HAQ, DAS28, CRP, and the calendar year of treatment start.

All data were analyzed using SPSS statistics 20.0 (SPSS Inc.). Level of significance was $\mathrm{p}<0.05$

\section{RESULTS}

Baseline characteristics for first analyzed treatment per patient. Demographic, disease, and patient characteristics at the start of anti-TNF therapy are presented in Table 1, stratified according to never remission (never DAS28<2.6), non-SR (DAS28 $<2.6$ for less than 6 mos), and SR (DAS28 $<2.6$ for $>6$ mos). Overall, the SR group had shorter disease duration and lower age, DAS28, and HAQ score.

Compared to ETN and ADA, patients treated with IFX had a higher MTX dose and fewer previous biologic treatments at anti-TNF start. There were no differences in DAS28 or disease duration between treatments; however, more men received IFX compared to ETN and ADA, and IFX-treated patients had higher HAQ scores.

Frequencies of SR for first SR treatment per patient. In total, 382 patients [15.8\%; 170 ETN (16.4\%), 133 IFX (14.2\%), and 79 ADA (17.8\%)] fulfilled the SR criteria. There were 133 patients $(34.8 \%)$ who escaped SR, i.e., had secondary failure. Of these 133, 115 continued the original treatment, 2 stopped, and 16 switched to another biologic. Altogether,
46 patients $(18.5 \%)$ stopped treatment at some time in the SR group. Twenty-one patients $(4.4 \%)$ were allowed to have DAS28 $\geq 2.6$ on 1 occasion given that they fulfilled the remission criteria for at least 6 months before and after. Five patients $(1.0 \%)$ were lost to followup after a mean of 55 months from treatment start in the SR group compared to 41 (1.4\%, nonsignificant difference) in the non-SR group (Figure 1).

Of the patients treated with concurrent MTX, 12.9\% had reached SR at 12 months compared to $5.4 \%$ of patients not treated with MTX. The percentage of patients receiving their first biologic treatment who reached SR was 16.1, compared to $11.0 \%$ of those with any previous biologic treatment. Fourteen patients had 2 SR episodes.

Predictors of SR. Figure 2 shows the predictors for achieving SR on first biologic treatment with OR and $95 \%$ CI. Age and female sex were both inversely related to achieving SR, as were HAQ score, DAS28, and the calendar year of treatment start. Concurrent MTX at baseline was positively associated with SR. PSL use and CRP did not predict SR. Of the 2311 biologic-naive patients in our registry, $203(8.8 \%)$ were excluded from the multivariate regression models because of the lack of followup data. There were no statistical differences in age, sex, disease duration, and concomitant DMARD usage between the excluded and those included in the regression models (data not shown). Cox regression model, taking time to remission into account, gave similar results.

Time to and duration of remission. Median estimated duration of SR for all patients was 63 months (5.25 yrs). The percentages of patients remaining in remission at 12,24 , and

Table 1. Demographic and clinical characteristics at baseline. Values are mean unless otherwise specified.

\begin{tabular}{|c|c|c|c|c|c|c|c|c|c|c|c|c|c|c|c|c|}
\hline \multirow[t]{2}{*}{ Characteristics } & \multicolumn{4}{|c|}{ All Patients } & \multicolumn{4}{|c|}{ Never Remission } & \multicolumn{4}{|c|}{ Non-sustained Remission } & \multicolumn{4}{|c|}{ Sustained Remission } \\
\hline & All & ETN & ADA & IFX & All & ETN & ADA & IFX & All & ETN & ADA & IFX & All & ETN & ADA & IFX \\
\hline $\mathrm{n}$ & 2416 & 1034 & 445 & 937 & 1222 & 534 & 230 & 458 & 812 & 317 & 136 & 359 & 382 & 170 & 79 & 133 \\
\hline$\%$ & 100 & 43 & 18 & 39 & 51 & 52 & 52 & 49 & 34 & 31 & 30 & 38 & 16 & 16 & 18 & 14 \\
\hline Age, yrs & 56.0 & 55.4 & 55.8 & 56.7 & 58.3 & 57.6 & 56.9 & 59.9 & 54.4 & 54.0 & 56.0 & 54.1 & 52.4 & 51.6 & 53.2 & 52.9 \\
\hline Female, $\%$ & 77 & 79 & 78 & 73 & 81 & 82 & 81 & 79 & 76 & 80 & 75 & 73 & 66 & 67 & 75 & 59 \\
\hline Disease duration, yrs & 11.8 & 12.1 & 10.5 & 12.2 & 12.7 & 12.8 & 10.5 & 13.6 & 11.5 & 11.9 & 11.2 & 11.2 & 10.1 & 10.4 & 9.8 & 9.9 \\
\hline HAQ & 1.3 & 1.3 & 1.2 & 1.4 & 1.4 & 1.4 & 1.3 & 1.6 & 1.3 & 1.3 & 1.2 & 1.3 & 1.0 & 1.0 & 1.0 & 1.0 \\
\hline DAS28 & 5.5 & 5.4 & 5.4 & 5.5 & 5.7 & 5.7 & 5.6 & 5.8 & 5.3 & 5.2 & 5.3 & 5.4 & 4.9 & 4.9 & 5.2 & 4.9 \\
\hline $\mathrm{CRP}, \mathrm{mg} / \mathrm{l}$ & 28.1 & 27.2 & 22.1 & 31.8 & 30.1 & 30.5 & 23.6 & 33.0 & 26.4 & 23.4 & 20.9 & 31.2 & 25.3 & 24.3 & 19.6 & 29.7 \\
\hline Previous DMARD, no. & 3.0 & 3.0 & 2.6 & 3.2 & 3.2 & 3.1 & 2.7 & 3.4 & 3.1 & 3.1 & 2.7 & 3.1 & 2.6 & 2.8 & 2.4 & 2.7 \\
\hline Previous biologic, $\%$ & 4.2 & 4.5 & 6.7 & 2.7 & 4.7 & 5.0 & 7.6 & 2.9 & 4.0 & 4.5 & 7.5 & 2.3 & 2.4 & 2.4 & 3.8 & 1.5 \\
\hline MTX at baseline, $\%$ & 61.4 & 54.0 & 62.7 & 68.9 & 55.6 & 50.3 & 54.2 & 62.6 & 63.9 & 55.3 & 66.2 & 70.7 & 77.0 & 66.5 & 81.0 & 88.0 \\
\hline MTX dose/week, mg & 16.0 & 17.1 & 17.1 & 14.7 & 15.4 & 16.7 & 17.4 & 13.3 & 16.2 & 16.8 & 16.7 & 15.6 & 17.2 & 18.3 & 16.9 & 16.3 \\
\hline PSL at baseline, $\%$ & 59.3 & 59.6 & 55.1 & 61.9 & 61.7 & 62.3 & 58.2 & 62.6 & 60.5 & 62.7 & 54.1 & 61.0 & 56.0 & 55.9 & 53.2 & 57.9 \\
\hline PSL, mg/day & 7.3 & 7.4 & 7.0 & 7.4 & 7.5 & 7.5 & 7.0 & 7.6 & 7.2 & 7.0 & 7.1 & 7.5 & 7.0 & 7.5 & 6.7 & 6.4 \\
\hline Ongoing DMARD, \% & 76.9 & 69.0 & 76.9 & 85.7 & 71.7 & 63.8 & 70.3 & 81.6 & 79.9 & 72.3 & 81.1 & 86.3 & 87.9 & 79.3 & 89.9 & 97.7 \\
\hline
\end{tabular}

DAS28 was based on the erythrocyte sedimentation rate level with 4 variables. Previous DMARD is the number of synthetic DMARD used previously at baseline. Ongoing DMARD is the percentage of patients taking any synthetic DMARD at baseline. ETN: etanercept; ADA: adalimumab; IFX: infliximab; HAQ: Health Assessment Questionnaire; DAS28: 28-joint Disease Activity Score; CRP: C-reactive protein; DMARD: disease-modifying antirheumatic drug; MTX: methotrexate; PSL: prednisolone.

Personal non-commercial use only. The Journal of Rheumatology Copyright (C) 2015. All rights reserved. 


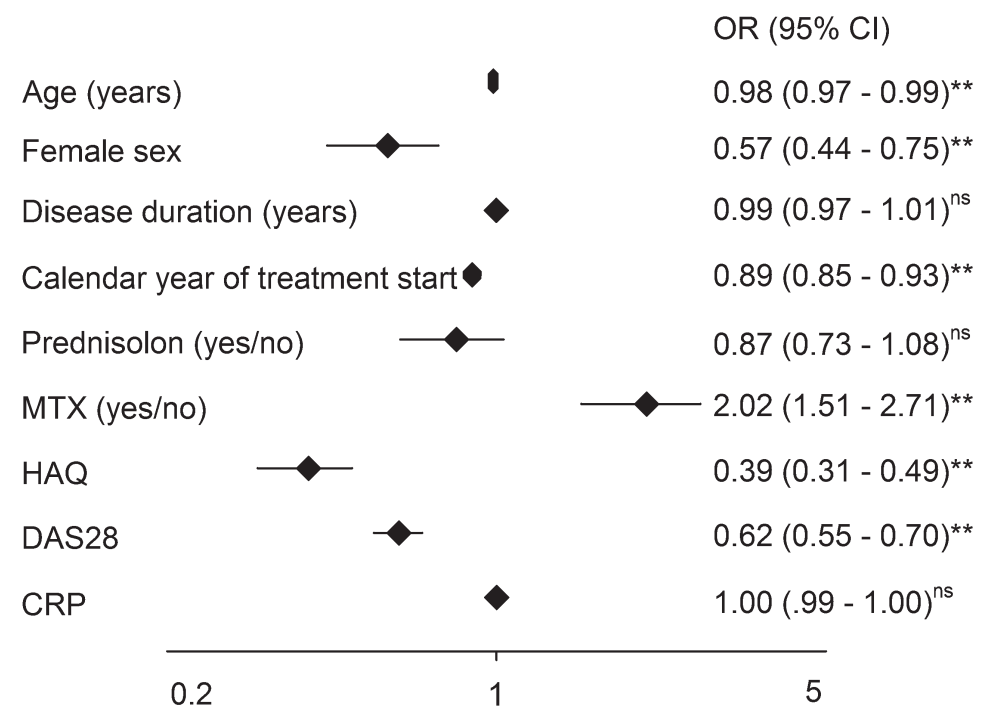

Figure 2. Predictors of sustained remission during first biologic treatment. Because of colinearity, separate models were used for DAS28 and HAQ, per unit increase, with the results from the HAQ model shown here. Female sex, male sex as a reference. DAS28 was based on the ESR level with 4 variables. $* \mathrm{p}<0.05$. $* * \mathrm{p}<0.005$. ns: not significant; MTX: methotrexate; HAQ: Health Assessment Questionnaire; DAS28: 28-joint Disease Activity Score; CRP: C-reactive protein; ESR: erythrocyte sedimentation rate.

48 months were $91.3 \%, 74.8 \%$, and $60.0 \%$, respectively. The longest remission in our cohort was 10.5 years. There were 254 patients $(66 \%)$ who stayed in remission for 24 months or more and $102(27 \%)$ who stayed for 48 months or more. Figure 3 shows the Kaplan-Meier survival curve for the percentage of treatments maintaining remission over time.

$A D A, E T N$, and IFX comparison (first biologic treatments

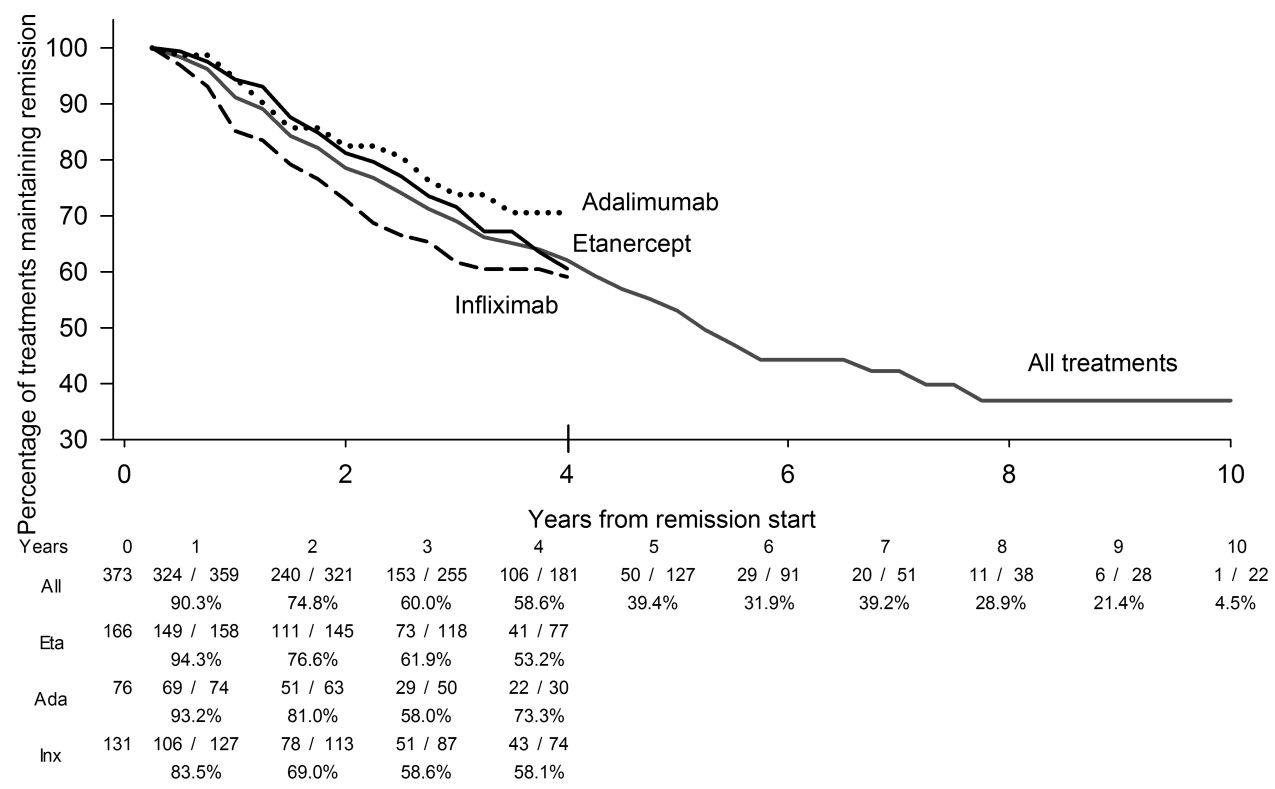

Figure 3. Kaplan-Meier survival curve for estimated remission duration (years). Percentage of treatments maintaining remission. The curves represent all anti-TNF treatments (grey line) and first biologic treatment with ADA (dotted), ETA (black line), and IFX (broken). Four-year followup time was used as a cutoff point for the drug comparison because ADA was not available in clinical practice until 2004. Beneath the figure are the number of subjects in remission for each subgroup and the number of patients still receiving treatment. For instance, after 5 years, 127 patients of those 373 at year 0 were still taking treatment, but 50 , or $39.4 \%$, were still in remission. ETA: etanercept; ADA: adalimumab; IFX: infliximab; anti-TNF: anti-tumor necrosis factor. 
only). Figure 4 shows the timing of SR onset in relation to treatment initiation. At 12 months, $11.9 \%$ of ETN-, $11.8 \%$ of ADA-, and $7.6 \%$ of IFX-treated patients fulfilled the SR criteria; the difference of ETN versus IFX $(\mathrm{p}<0.05)$ and ADA versus IFX being significant (Figure 4). After LUNDEX correction, $8.8 \%$ of ETN-treated patients, $7.2 \%$ of ADA-treated patients, and 5.3\% of IFX-treated patients had achieved SR within the first 12 months of treatment (Figure 4).

Adjusted OR for achieving SR within the first 12 months was 1.86 for ETN (95\% CI 1.33-2.61), with IFX as the reference drug. ADA was not significantly different from ETN or IFX (Figure 5). Adjusted HR for achieving SR within the first 4 years of treatment were 1.32 for ETN (95\% CI 1.01-1.74) and 1.84 for ADA (95\% CI 1.23-2.78), with IFX as the reference drug (Figure 5).

Adjusted HR for continued SR for 4 years without secondary failure was 1.93 for ADA (95\% CI 1.06-3.50) with IFX as the reference drug, while ETN was not significantly different from ADA or IFX (Figure 5).

\section{DISCUSSION}

SR was uncommon in this large population-based cohort of anti-TNF-treated patients with RA managed in daily clinical practice. However, patients who reached SR remained in remission for a substantial period of time, with a median of
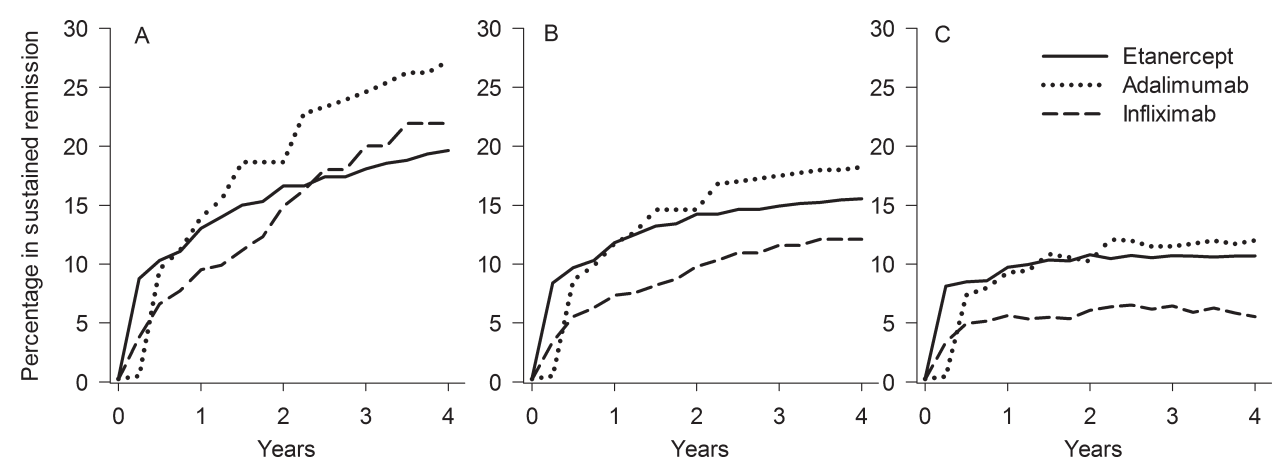

Figure 4. Inverted Kaplan-Meier survival curves showing timing (years) of remission onset in relation to treatment initiation for first biologic treatment. A. Per protocol method. B. Intention to treat using the last observation carried forward method. C. LUNDEX correction. Four-year followup time was used as a cutoff point for the drug comparison because adalimumab was not available in clinical practice until 2004.

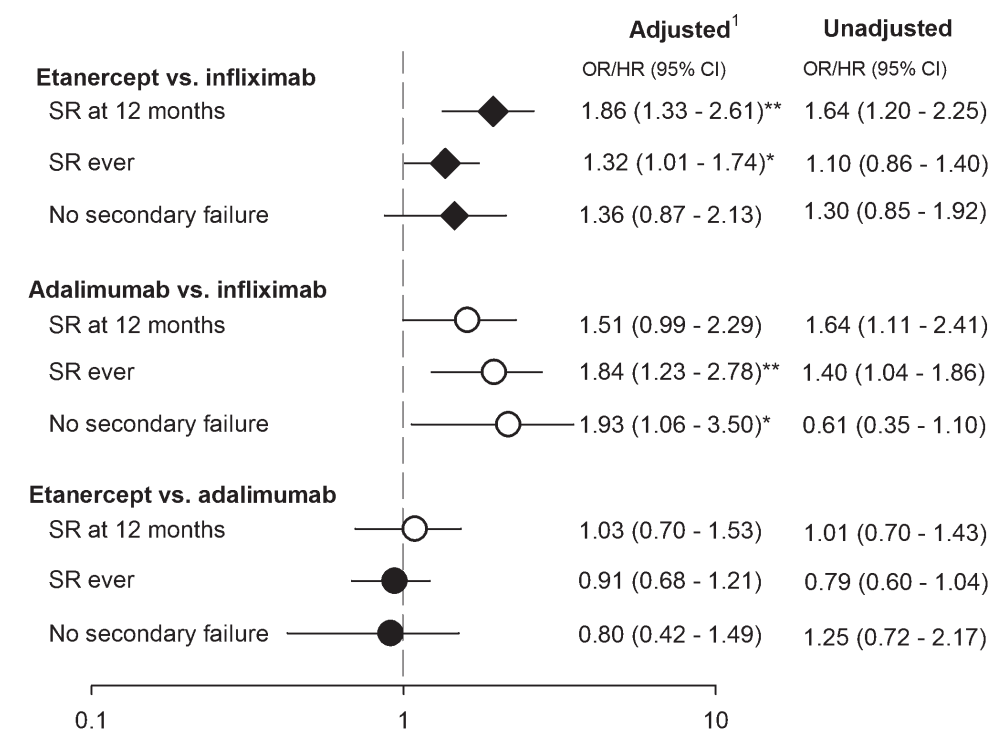

Figure 5. Odds of having achieved SR with first biologic after 12 months. HR for achieving SR with first biologic in the first 4 years of treatment and HR for continued remission without secondary failure within 4 years. Higher OR/HR favors drug to the left. On the right the results for the unadjusted analyses are presented. $* \mathrm{p}<0.05 . * * \mathrm{p}<0.005 .{ }^{1}$ Adjusted for those baseline variables that significantly changed the unadjusted or raw model. SR: sustained remission. 
over 5 years. Concomitant MTX treatment, younger age, male sex, low disease activity, and good functional capacity were identified as positive predictors of SR. Head-to-head comparison between individual anti-TNF drugs revealed that ETN and ADA were more likely to bring about SR than was IFX.

Only $15.8 \%$ of initiated anti-TNF treatments resulted in remission lasting 6 months or longer with a median remission time of 63 months. The fact that 159 patients of the 189 who escaped SR have continued with the same treatment indicates that patients most often go from remission to low disease activity, and that the prescribing physician or patient did not consider the increased activity a sufficient reason to discontinue treatment.

Criteria for SR have not been firmly established, and our time frame of at least a 6-month duration is arbitrary but reasonably practical when it comes to patients in the observational setting because 6-month intervals are usually part of the SSATG followup schedule during the first 2 years, and thereafter at least once a year. Furst, et $a l^{16}$ defined sustained DAS28 remission as remission during any 2 consecutive visits within 1 year after treatment initiation. In an observational study of a clinical cohort in the United States, $7.9 \%$ reached SR in the first 12 months compared to $10.1 \%$ in our cohort. In an analysis from a US-based cohort of patients with RA in clinical practice (even those without biologics), only $41 \%$ of those reaching remission were still in remission at 12 months, with a median remission time of 1 year $^{17}$. Shahouri, et $a l^{18}$ also examined data from US and Canadian clinical practices and observed that within 12 months, $65-80 \%$ of those patients who had experienced remission no longer met remission criteria, and at 24 months, only $6-14 \%$ still met remission criteria. Because the duration of followup in our cohort differs (0-149 months), we performed a survival analysis of remission time. More than half of those reaching SR were still in remission after 24 months and the median estimated duration of remission for all treatments was 63 months. This means that once in remission, patients treated with anti-TNF will remain in remission for a substantial period of time. It must be remembered that different criteria sets for remission were used and the different healthcare settings preclude head-to-head comparisons.

To study the probability for SR, a multivariate logistic regression analysis was used. However, because the time period to SR could have had a clinically relevant effect on SR, we also performed a more explorative approach with a multivariate Cox regression model, taking into account time to reach this outcome. The results were similar irrespective of method chosen, but our findings should be validated in other patient populations.

An important finding in our study was that low HAQ, reflecting high functional ability and low disability, was a strong predictor of SR. Other predictors of SR were concomitant MTX usage and lower DAS28 score at treatment initiation, while female sex and higher age were inversely related to SR. A systematic review of prognostic studies that identifies the predictors of remission in patients with RA found a number of variables to be independent predictors of RA remission. These included male sex, young age, late-onset RA, short disease duration, low baseline disease activity, and early treatment with combination of DMARD $^{19}$. Our results are consistent with analyses on response to biologic treatment in the registries in southern Sweden $^{20}$, Germany ${ }^{21}$, and Britain ${ }^{22}$. Others have also reported that shorter disease duration was a predictor for reaching $\mathrm{SR}^{16}$. This was not true in our study. Our results suggest that disability rather than disease duration is the predictive factor for SR. Level of disability has been shown to be directly related to HAQ score in established $\mathrm{RA}^{23}$. Male sex has been shown to be a predictive factor for DAS28 remission ${ }^{24}$ and for a response to biologic treatment $^{20,22}$, but it has been disputed whether men truly respond better to treatment or whether the difference lies in the tools used to measure disease activity ${ }^{25,26}$.

There are few studies to date that have attempted to compare the efficacy of different anti-TNF treatments, and formal head-to-head RCT are rare. In the absence of superiority studies, we had to use data from metaanalyses and registries. Cohorts in randomized controlled studies reflected only a minor proportion of the patients treated with biologic agents in routine care; only about one-fourth of the patients in the German biologics register would have been eligible for the major trials ${ }^{27}$. Hetland, et $a l^{28}$ have published data from the Danish registry directly comparing ETN, ADA, and IFX responses and remission rates. In agreement with our study, IFX was associated with the lowest crude rates of treatment response and disease remission at 12 months. As in our study, the IFX-treated patients were often scored on the day of their infusion. An observational study from a register in the Netherlands compared ADA, ETN, and IFX effectiveness in reducing DAS28 at 12 months compared to baseline. The decrease was significantly larger for ADA and ETN in comparison to IFX, but the results did not include remission rates ${ }^{29}$.

In our drug comparison, we focused on patients that had not received any biologic treatment earlier. Biologic-naive patients treated with ETN had significantly better SR rates and OR for achieving SR at 12 months than IFX-treated patients. After the first 6 months of treatment, IFX had a lower LUNDEX value. The LUNDEX is a valuable tool for evaluating drug efficacy in observational studies. Calculating the proportion of patients who continue therapy multiplied by the proportion of patients who fulfill the criteria for SR at a given time, LUNDEX takes into account all patients who discontinued treatment before that timepoint. Thus it provides useful information on the percentage of all patients who initiated treatment and actually reached

Personal non-commercial use only. The Journal of Rheumatology Copyright @ 2015 . All rights reserved. 
remission ${ }^{15}$. Actually, LUNDEX corresponds to nonresponder imputation of dropouts in RCT, i.e., the most conservative interpretation.

We chose DAS28 remission criteria as a definition for clinical remission. This is the most widely used definition in trials during the last decade. It has been suggested that patients in DAS28 remission represent minimal disease activity rather than true disease-free state ${ }^{9,23,30}$. The ACR/EULAR criteria presented in $2011^{8}$ are stricter and are mostly considered criteria for clinical trials in early arthritis; therefore they are less applicable in patients with longstanding disease. Our patients had an average disease duration of almost 12 years. Assessing disease activity in such a group is problematic because joint swelling and tenderness may be a consequence of irreversible structural damage that also leads to a higher score on the visual analog scales.

Our analysis supports the combined use of MTX and anti-TNF therapy, with more than twice as many patients in remission at 12 months compared to those without MTX treatment. The long disease duration probably explains why so few are treated with concomitant MTX. Almost every patient had been treated earlier with synthetic DMARD that had been stopped, and thus represents a subset of patients with RA who are either nonresponsive to or intolerant of 1 or several traditional DMARD. Similar figures are found in the North American registry, where only $67.7 \%$ of patients with longstanding disease were treated with concomitant MTX $^{16}$.

As opposed to several other register studies, we know from 2 different validation studies that the coverage of the SSATG is almost complete regarding biologic drugs prescribed to patients with arthritis in the area of southern Sweden, and the results can thus be regarded as population-based for these drugs in patients with arthritis. An additional strength of our study is the search process. When searching for treatments fulfilling the SR criteria, every case was thoroughly scrutinized; researchers were able to look at the whole treatment period graphically and then look at every visit in detail, as opposed to just a simple database search. This made possible inclusion of occasional followup visits not fulfilling the criteria in the SR periods. This is an important part of the clinical practice setting where a patient may well be evaluated for their arthritis, but is also being treated for a comorbid condition such as an infection that will have a short-lived effect on the SR measures. The SSATG has a structured followup protocol that has been described earlier ${ }^{11}$, but in an open cohort like ours, patients come on extra visits as needed and sometimes miss their visits. The nonrandomized, open nature of observational studies generates methodological limitations ${ }^{20,31,32}$. Decisions to start or stop therapy with a certain agent were based solely on clinical practice and the experience of treating physicians with national and international guidelines as support. Variations in baseline data indicate that TNF inhibitors are used differently. Although we have corrected for baseline characteristics, confounding by indication, and channeling, observation bias or other unmeasured confounders such as radiographic changes, comorbidity, or compliance cannot be completely ruled out. We do not have complete data on baseline differences in comorbidities, but earlier linkage to an in-hospital discharge register with the same patient cohort showed no baseline difference in prior diabetes, malignancies, chronic pulmonary diseases, and cardiovascular diseases when comparing patients treated with ETN or IFX ${ }^{33}$. Although these methodological limitations should be borne in mind when interpreting the results, observational studies remain important in offering information from daily clinical practice.

SR was not common in patients with RA receiving anti-TNF in the observational SSATG setting. However, patients with RA treated with anti-TNF who achieved 6 months of DAS28 remission remained in remission for a substantial period of time. Male sex, lower age, low DAS and HAQ, and concomitant MTX were positive predictors of SR. Differences were seen in remission rates and duration between different medications. Within 12 months, ETN had a significantly higher likelihood of bringing about remission compared to IFX, which had significantly higher rates of secondary failure than ADA.

\section{ACKNOWLEDGMENT}

We thank Jan-Åke Nilsson for his valuable and constructive criticism.

\section{REFERENCES}

1. Cooper NJ. Economic burden of rheumatoid arthritis: a systematic review. Rheumatology 2000;39:28-33.

2. Zhang W, Anis AH. The economic burden of rheumatoid arthritis: beyond health care costs. Clin Rheumatol 2011;30 Suppl 1:S25-32.

3. Kvien TK. Epidemiology and burden of illness of rheumatoid arthritis. Pharmacoeconomics 2004;22 Suppl 1:1-12.

4. Pincus T, Callahan LF. What is the natural history of rheumatoid arthritis? Rheum Dis Clin North Am 1993;19:123-51.

5. Genovese MC. Treatment of rheumatoid arthritis. In: Firestein GS, Budd RC, Harris ED Jr., McInnes IB, Ruddy S, Sergent JS, eds. Kelley's textbook of rheumatology, 8th ed. Philadelphia: Saunders; 2008.

6. Smolen JS, Aletaha D, Bijlsma JW, Breedveld FC, Boumpas D, Burmester G, et al. Treating rheumatoid arthritis to target: recommendations of an international task force. Ann Rheum Dis 2010;69:631-7.

7. Smolen JS, Landewé R, Breedveld FC, Dougados M, Emery P, Gaujoux-Viala C, et al. EULAR recommendations for the management of rheumatoid arthritis with synthetic and biological disease-modifying antirheumatic drugs. Ann Rheum Dis 2010;69:964-75.

8. Felson DT, Smolen JS, Wells G, Zhang B, van Tuyl LH, Funovits J, et al. American College of Rheumatology/European League against Rheumatism provisional definition of remission in rheumatoid arthritis for clinical trials. Ann Rheum Dis 2011;70:404-13.

9. van der Heijde D, Klareskog L, Boers M, Landewé R, Codreanu C, Bolosiu HD, et al. Comparison of different definitions to classify remission and sustained remission: 1 year TEMPO results. Ann

Personal non-commercial use only. The Journal of Rheumatology Copyright (c) 2015. All rights reserved. 
Rheum Dis 2005;64:1582-7.

10. Smolen JS, Han C, van der Heijde DM, Emery P, Bathon JM, Keystone E, et al. Radiographic changes in rheumatoid arthritis patients attaining different disease activity states with methotrexate monotherapy and infliximab plus methotrexate: the impacts of remission and tumour necrosis factor blockade. Ann Rheum Dis 2009;68:823-7.

11. Geborek P, Saxne T. Clinical protocol for monitoring of targeted therapies in rheumatoid arthritis. Rheumatol 2000;39:1159-61.

12. Geborek P, Nitelius E, Noltorp S, Petri H, Jacobsson L, Larsson L, et al. Population based studies of biological antirheumatic drug use in southern Sweden: comparison with pharmaceutical sales. Ann Rheum Dis 2005;64:1805-7.

13. Neovius M, Simard J, Sundström A, Jacobsson L, Geborek P, Saxne $\mathrm{T}$, et al. Generalisability of clinical registers used for drug safety and comparative effectiveness research: coverage of the Swedish Biologics Register. Ann Rheum Dis 2011;70:516-9.

14. Geborek P, Crnkic M, Petersson IF, Saxne T; South Swedish Arthritis Treatment Group. Etanercept, infliximab, and leflunomide in established rheumatoid arthritis: clinical experience using a structured follow up programme in southern Sweden. Ann Rheum Dis 2002;61:793-8.

15. Kristensen LE, Saxne T, Geborek P. The LUNDEX, a new index of drug efficacy in clinical practice: results of a five-year observational study of treatment with infliximab and etanercept among rheumatoid arthritis patients in southern Sweden. Arthritis Rheum 2006;54:600-6.

16. Furst DE, Pangan AL, Harrold LR, Chang H, Reed G, Kremer JM, et al. Greater likelihood of remission in rheumatoid arthritis patients treated earlier in the disease course: results from the Consortium of Rheumatology Researchers of North America registry. Arthritis Care Res 2011;63:856-64.

17. Prince FH, Bykerk VP, Shadick NA, Lu B, Cui J, Frits M, et al. Sustained rheumatoid arthritis remission is uncommon in clinical practice. Arthritis Res Ther 2012;14:R68.

18. Shahouri SH, Michaud K, Mikuls TR, Caplan L, Shaver TS, Anderson JD, et al. Remission of rheumatoid arthritis in clinical practice: application of the American College of Rheumatology/European League Against Rheumatism 2011 remission criteria. Arthritis Rheum 2011;63:3204-15.

19. Katchamart W, Johnson S, Lin HJ, Phumethum V, Salliot C, Bombardier C. Predictors for remission in rheumatoid arthritis patients: a systematic review. Arthritis Care Res 2010;62:1128-43.

20. Kristensen LE, Kapetanovic MC, Gülfe A, Söderlin M, Saxne T, Geborek P. Predictors of response to anti-TNF therapy according to ACR and EULAR criteria in patients with established RA: results from the South Swedish Arthritis Treatment Group Register. Rheumatology 2008;47:495-9.

21. Listing J, Strangfeld A, Rau R, Kekow J, Gromnica-Ihle E, Klopsch $\mathrm{T}$, et al. Clinical and functional remission: even though biologics are superior to conventional DMARDs overall success rates remain low - results from RABBIT, the German biologics register. Arthritis Res Ther 2006;8:R66.
22. Hyrich KL, Watson KD, Silman AJ, Symmons DP; British Society for Rheumatology Biologics Register. Predictors of response to anti-TNF-alpha therapy among patients with rheumatoid arthritis: results from the British Society for Rheumatology Biologics Register. Rheumatology 2006;45:1558-65.

23. Aletaha D, Ward MM, Machold KP, Nell VP, Stamm T, Smolen JS. Remission and active disease in rheumatoid arthritis: defining criteria for disease activity states. Arthritis Rheum 2005; 52:2625-36.

24. Forslind K, Hafström I, Ahlmén M, Svensson B; BARFOT Study Group. Sex: a major predictor of remission in early rheumatoid arthritis? Ann Rheum Dis 2007;66:46-52.

25. Mäkinen H, Hannonen P, Sokka T. Sex: a major predictor of remission as measured by 28-joint Disease Activity Score (DAS28) in early rheumatoid arthritis? Ann Rheum Dis 2008;67:1052-3.

26. Ahlmén M, Svensson B, Albertsson K, Forslind K, Hafström I; BARFOT Study Group. Influence of gender on assessments of disease activity and function in early rheumatoid arthritis in relation to radiographic joint damage. Ann Rheum Dis 2010;69:230-3.

27. Zink A, Strangfeld A, Schneider M, Herzer P, Hierse F, Stoyanova-Scholz M, et al. Effectiveness of tumor necrosis factor inhibitors in rheumatoid arthritis in an observational cohort study: comparison of patients according to their eligibility for major randomized clinical trials. Arthritis Rheum 2006;54:3399-407.

28. Hetland ML, Christensen IJ, Tarp U, Dreyer L, Hansen A, Hansen IT, et al. Direct comparison of treatment responses, remission rates, and drug adherence in patients with rheumatoid arthritis treated with adalimumab, etanercept, or infliximab: results from eight years of surveillance of clinical practice in the nationwide Danish DANBIO registry. Arthritis Rheum 2010;62:22-32.

29. Kievit W, Adang EM, Fransen J, Kuper HH, van de Laar MA, Jansen TL, et al. The effectiveness and medication costs of three anti-tumour necrosis factor alpha agents in the treatment of rheumatoid arthritis from prospective clinical practice data. Ann Rheum Dis 2008;67:1229-34.

30. Wells GA, Boers M, Shea B, Brooks PM, Simon LS, Strand CV, et al. Minimal disease activity for rheumatoid arthritis: a preliminary definition. J Rheumatol 2005;32:2016-24.

31. Pincus T, Sokka T. Should contemporary rheumatoid arthritis clinical trials be more like standard patient care and vice versa? Ann Rheum Dis 2004;63 Suppl 2:ii32-9.

32. Wolfe F, Michaud K, Dewitt EM. Why results of clinical trials and observational studies of antitumour necrosis factor (anti-TNF) therapy differ: methodological and interpretive issues. Ann Rheum Dis 2004;63 Suppl 2:ii13-7.

33. Kristensen LE, Saxne T, Nilsson JA, Geborek P. Impact of concomitant DMARD therapy on adherence to treatment with etanercept and infliximab in rheumatoid arthritis. Results from a six-year observational study in southern Sweden. Arthritis Res Ther 2006;8:R174. 